The candidate gene approach in asthma: what happens with the neighbours?

European Journal of Human Genetics (2010) 18, 17; doi:10.1038/ejhg.2009.128; published online 5 August 2009

In the last few decades, multiple genes important in asthma and atopy development have been identified. A successful approach has been to investigate candidate genes, that is, genes with a biologically plausible function. ${ }^{1}$ This approach has also been applied by Zhu et $a l^{2}$ in a previous issue of this journal. They analysed IL18RI, an interesting candidate gene for asthma and atopy, and provided replicated evidence in three European populations that SNPs located in IL18R1 were associated with asthma. ${ }^{2}$ Specifically, SNP rs1420099, rs1362348, and rs1974657 were associated with asthma in these three populations.

IL-18 receptor is a key immunoregulator; the gene product of IL18R1 forms the alpha chain of the IL-18 receptor. ${ }^{3}$ Binding of IL-18 to the IL-18 receptor can stimulate Th1 as well as Th2 cytokine release. ${ }^{4}$ These findings may indeed point towards a role of IL18R1 in the pathophysiology of asthma. ${ }^{2}$ IL18R1 is localized in the IL1 receptor cluster on chromosome $2 \mathrm{q} 12$. In its close vicinity reside IL1R2, IL1RL, IL1RL2, IL1RL1, and IL18RAP. We have recently undertaken a candidate gene approach analysing genes located in a region of strong linkage disequilibrium (LD) in this gene cluster, that is, IL18R1, as well as ILIRLI and IL18RAP, in two Dutch asthma and one Dutch rhinitis cohort. ${ }^{5}$ We reported replicated evidence for association of SNPs in this gene cluster with asthma phenotypes in our two Dutch asthma populations. For IL18R1, four SNPs were associated with asthma and bronchial hyperresponsiveness in a combined analysis of the two asthma cohorts $(P<0.05)$; these SNPs, that is, rs12999364, rs1558627, rs2270297, and rs1035130, were not genotyped by Zhu et al. Furthermore, we found significant associations with SNPs in IL1RL1 and IL18RAP. A haplotype from SNPs in IL1RL1 and IL18R1 was significantly associated with bronchial hyperresponsiveness. Strong LD was detected between SNPs in the three genes in this region.

IL1RL1 encodes the receptor for IL-33, which is located on mast cells, Th2 cells, regulatory T cells, and macrophages, and is also present in serum in a soluble form..$^{6-8}$ IL1RL1 is a member of the Toll-like receptor superfamily and can either stimulate or inhibit Th2 responses by influencing TLR pathway signalling. ${ }^{9-12}$ There is increasing evidence that this gene is important in atopic diseases such as eczema and asthma; interestingly, a recent large genome-wide association study also indicated ILIRL1 to be important in asthma, and thus IL1RL1 is also a plausible candidate gene for asthma., 5,14

In their paper, Zhu et al mentioned the limitation of not analysing SNPs located in IL18R2 (also known as IL18RAP). SNPs in IL1RL1 and IL18RAP, next to IL18R1, may also contribute to the genetic association signal on chromosome $2 \mathrm{q} 12$. We suggest that genetic association studies in regions with strong LD may not be conclusive as to which gene or genes are causal in disease development. It would therefore be of interest to investigate also IL1RL1 and IL18RAP in the populations described by Zhu et al. Moreover, we suggest the investigation of this region in populations with different LD characteristics and to perform functional studies. Our observations imply that, once positive genetic associations are identified, it is worthwhile to take a look at the neighbouring genes.

Naomi E Reijmerink ${ }^{1,2}$, Dirkje S Postma ${ }^{1}$ and Gerard H Koppelman ${ }^{3}$ ${ }^{1}$ Department of Pulmonology, University Medical Center Groningen, University of Groningen, Groningen, The Netherlands; ${ }^{2}$ Department of Pediatrics, Beatrix Children's Hospital, University Medical Center Groningen,

University of Groningen, Groningen, The Netherlands; ${ }^{3}$ Department of Pediatric Pulmonology and Pediatric Allergology, Beatrix Children's Hospital, University Medical Center Groningen, University of Groningen, Groningen, The Netherlands E-mail: g.h.koppelman@bkk.umcg.nl

1 Vercelli D: Discovering susceptibility genes for asthma and allergy. Nat Rev Immunol 2008; 8: 169-182.

2 Zhu G, Whyte MK, Vestbo J et al: Interleukin 18 receptor 1 gene polymorphisms are associated with asthma. Eur J Hum Genet 2008; 16: 1083-1090.

3 Torigoe K, Ushio S, Okura T et al: Purification and characterization of the human interleukin-18 receptor. J Biol Chem 1997; 272: 25737-25742.

4 Nakanishi K, Yoshimoto T, Tsutsui H, Okamura H: Interleukin-18 is a unique cytokine that stimulates both Th1 and Th2 responses depending on its cytokine milieu. Cytokine Growth Factor Rev 2001; 12: 53-72.

5 Reijmerink NE, Postma DS, Bruinenberg M et al: Association of IL1RL1, IL18R1, and IL18RAP gene cluster polymorphisms with asthma and atopy. J Allergy Clin Immunol 2008; 122: 651-654.

$6 \mathrm{Xu} \mathrm{D}$, Chan WL, Leung BP et al: Selective expression of a stable cell surface molecule on type 2 but not type 1 helper T cells. J Exp Med 1998; 187: 787-794.

7 McGuirk P, McCann C, Mills KH: Pathogen-specific T regulatory 1 cells induced in the respiratory tract by a bacterial molecule that stimulates interleukin 10 production by dendritic cells: a novel strategy for evasion of protective T helper type 1 responses by Bordetella pertussis. J Exp Med 2002; 195: 221-231.

8 Lecart S, Lecointe N, Subramaniam A et al: Activated. but not resting human Th2 cells, in contrast to Th1 and T regulatory cells, produce soluble ST2 and express low levels of ST2L at the cell surface. Eur J Immunol 2002; 32: 2979-2987.

9 Hayakawa H, Hayakawa M, Kume A, Tominaga S: Soluble ST2 blocks interleukin-33 signaling in allergic airway inflammation. J Biol Chem 2007; 282: 26369-26380.

10 Brint EK, Xu D, Liu H et al: ST2 is an inhibitor of interleukin 1 receptor and Toll-like receptor 4 signaling and maintains endotoxin tolerance. Nat Immunol 2004; 5: 373-379.

11 Schmitz J, Owyang A, Oldham E et al: IL-33. an interleukin-1-like cytokine that signals via the IL-1 receptor-related protein ST2 and induces T helper type 2-associated cytokines. Immunity 2005; 23: 479-490.

12 Mangan NE, Dasvarma A, McKenzie AN, Fallon PG: T1/ST2 expression on Th2 cells negatively regulates allergic pulmonary inflammation. Eur J Immunol 2007; 37: 1302-1312.

13 Gudbjartsson DF, Bjornsdottir US, Halapi E et al: Sequence variants affecting eosinophil numbers associate with asthma and myocardial infarction. Nat Genet 2009; 41: 342-347.

14 Shimizu M, Matsuda A, Yanagisawa K et al: Functional SNPs in the distal promoter of the ST2 gene are associated with atopic dermatitis. Hum Mol Genet 2005; 14: 2919-2927.

\section{Reply to Reijmerink et al}

European Journal of Human Genetics (2010) 18, 17-18; doi:10.1038/ejhg.2009.130; published online 5 August 2009

We appreciate the comments from Reijmerink et al on our IL18R1 genetic association results published in the European Journal of Human Genetics. ${ }^{1}$ As we had pointed out in the paper, ours was a 
candidate gene study specifically exploring the hypothesis of genetic association between IL18R1 and asthma, as IL18R1 is a plausible candidate based on the available biological information. The observation that the SNPs in IL1RL1, IL18RAP and IL18R1 are associated with asthma in the Dutch asthma cohorts is interesting. We did not discuss the possibility of association with this gene cluster in our paper, as we were focused on IL18R1's association with asthma.

A recent GWAS study to identify sequence variants affecting blood eosinophil counts reported significant association with the LD block containing IL1RL1, IL18R1, IL18RAP and SLC9A4, and this region is significantly associated with asthma $\left(P=5.5 \times 10^{-12}\right)$ in a collection of 10 different populations. ${ }^{2}$ This study, along with the results pointed out by Reijmerink et $a l^{3}$ and our study, ${ }^{1}$ highlights the importance of this region in asthma-related phenotypes. As IL1RL1, IL18R1, IL18RAP and SLC9A4 are in tight linkage disequilibrium, we agree with Reijmerink et al's suggestion to take a closer look at this region in large asthma populations. Considering the LD pattern in this gene cluster, genotyping and analyzing additional SNPs may not resolve the questions raised here. We suggest deep sequencing of this region in a large population to identify functional variants and also suggest undertaking functional studies to pinpoint the biological association with the disease. We agree with the comments of Reijmerink et al on the significance of this region and highlight the importance of examining the flanking regions in all candidate gene studies. Recent efforts in setting up large databases for genetic association studies in asthma (GABRIEL consortium) and other diseases will be helpful to solve some of these questions.

Sreekumar G Pillai ${ }^{*, 1}$

${ }^{1}$ Genetics, GlaxoSmithKline R\&D, Research Triangle Park, NC, USA ${ }^{*}$ Correspondence: Dr S Pillai, Genetics, GlaxoSmithKline R\&D, Research Triangle Park, NC 27709, USA. Tel: +1 919483 1815; Fax: +1 919315 0311; E-mail: Pillaisreekumar@yahoo.com or sreekumar.g.pillai@gsk.com

1 Zhu G, Whyte MK, Vestbo J et al: Interleukin 18 receptor 1 gene polymorphisms are associated with asthma. Eur J Hum Genet 2008; 16: 1083-1090.

2 Gudbjartsson DF, Bjornsdottir US, Halapi E et al: Sequence variants affecting eosinophil numbers associate with asthma and myocardial infarction. Nat Genet 2009; 41: 342-347.

3 Reijmerink NE, Postma DS, Bruinenberg M et al: Association of IL1RL1, IL18R1, and IL18RAP gene cluster polymorphisms with asthma and atopy. J Allergy Clin Immunol 2008; 122: 651-654. 